\title{
Seguimiento de la calidad de un embalse de abastecimiento de agua potable según las directrices de la Directiva Marco (embalse del Añarbe. Cuenca Norte)
}

\author{
Henar Fraile ${ }^{1}$, José Manuel Leonardo ${ }^{1}$, Begoña G. de Bikuña ${ }^{1}$ e Itziar Larumbe ${ }^{2}$ \\ 1 Anbiotek S.L. Ribera de Axpe 11, B-201 48950 Erandio. \\ 2 AGASA P $^{o}$ de Errotaburu 1-6 ${ }^{a} 20018$ Donostia \\ * Autor responsable de la correspondencia: henar@anbiotek.com
}

Recibido: 2/11/06

Aceptado: $11 / 4 / 08$

\begin{abstract}
Monitoring of the quality of a drinking water supply reservoir according to the Water Framework Directive (Añarbe reservoir, North basin)

The Añarbe reservoir is located in the Iberic-Macaronesian region and is a water body provisionally identified as heavily modified, comparable to "lake', that according to the "A" classification system of the WFD is "siliceous, lowlands, big and very deep", and according to the "B" system is "lowlands, northern, warm monomictic and of acidic waters". Two annual cycles of studies have been completed (from May of 2004 to January of 2006) looking to the main biological, physicochemical and hydromorphological parameters. The trophic state is oligotrophic for most of the parameters, except for the total phosphorus, that classifies it as mesotrophic. It does not present algal blooms and the average oxygen concentration in the hypolimnion in the zone of the dam during the stratification is $4.5 \mathrm{mg} \mathrm{O}_{2} / 1$. The percentage of variation of the water volume of the reservoir respective to the maximum volume varies between 40 and $33 \%$ for the years 2004 and 2005, respectively. It shows a good ecological potential and could be considered as a reference within its group.
\end{abstract}

Key words: Reservoir, trophic state, ecological potential.

\section{RESUMEN}

Seguimiento de la calidad de un embalse de abastecimiento de agua potable según las directrices de la Directiva Marco (embalse del Añarbe. Cuenca Norte)

El embalse del Añarbe se encuentra en la Región ibérico-macaronésica y es una masa de agua identificada provisionalmente como muy modificada, asimilable a 'lago', que según el Sistema A de clasificación de la DMA es del tipo 'silíceo, tierras bajas, grande y muy profundo' y según el Sistema B; 'tierras bajas, septentrional, monomíctico cálido y de aguas ácidas'. Se han completado dos ciclos anuales de estudio (desde mayo de 2004 hasta enero de 2006) con los principales parámetros biológicos, fisicoquímicos e hidromorfológicos. Su estado trófico es de oligotrofia para la mayoría de parámetros, excepto para el fósforo total, que lo clasifica como mesotrófico. No presenta blooms algales y la concentración media de oxígeno en el hipolimnion de la zona de la presa durante la estratificación es de $4.5 \mathrm{mg} \mathrm{O}_{2} / \mathrm{l}$. El porcentaje de variación del volumen del embalse con respecto al volumen máximo varía entre el 40 y el $33 \%$, para los años 2004 y 2005, respectivamente. Presenta un buen potencial ecológico y podría ser considerado como de referencia para su tipología.

Palabras clave: Embalse, estado trófico, potencial ecológico. 


\section{INTRODUCCIÓN}

Uno de los elementos más novedosos de la Directiva marco del agua (2000/60/CE) (DOCE, 2000), en adelante DMA, es la concepción del término 'estado ecológico' que se utiliza para expresar la calidad de la estructura y funcionamiento de los ecosistemas acuáticos asociados a las aguas superficiales. En el caso de masas de agua artificiales o muy modificadas se utiliza el término 'potencial ecológico', que en el mejor de los casos se corresponde con un potencial ecológico máximo (MPE), ya que las condiciones naturales muy buenas u óptimas se han perdido.

Para definir el estado o potencial ecológico es necesario considerar tres criterios básicos (WFD CIS $\mathrm{N}^{\circ}$ 4, 2003; WFD CIS $\mathrm{N}^{\circ}$ 10, 2003; WFD CIS No 13, 2005):

- Identificar las masas de agua superficiales, encuadrándolas dentro de una de las categorías definidas en la DMA (ríos, lagos, aguas de transición y aguas costeras) o bien como masas de agua artificiales o muy modificadas,

- Tipificar dichas masas de agua según el sistema A o bien el B de la DMA y establecer unas condiciones de referencia acordes a las características de las mismas,

- Definir el estado o potencial ecológico como una expresión integrada de la diferencia existente entre los valores de los indicadores biológicos, fisicoquímicos e hidromorfológicos evaluados, frente a los valores que, para esos mismos indicadores, se han establecido en las condiciones de referencia.

El principal objetivo de la DMA es lograr alcanzar un buen estado o potencial ecológico en las masas de agua para el horizonte del año 2015.

La DMA establece que las masas de agua utilizadas para la captación de agua destinada al consumo humano que proporcionen un promedio de más de $100 \mathrm{~m}^{3}$ diarios deberán ser controladas mediante un seguimiento de sus indicadores biológicos, hidromorfológicos y fisicoquímicos según se describe en su Anexo V. En este contexto el organismo gestor del embalse del Añar- be (AGASA) ha promovido desde la primavera de 2004 hasta la fecha, la realización de una serie de controles realizados de manera conjunta por la empresa Anbiotek S.L. y el laboratorio de AGASA. En este trabajo se resume el estudio de dos ciclos anuales en el embalse del Añarbe desde un punto de vista limnológico encaminado hacia la definición de su estado trófico, pero incluyendo además como elemento novedoso una aproximación metodológica al cálculo del potencial ecológico siguiendo las directrices de la DMA.

\section{ÁREA DE ESTUDIO}

El embalse del Añarbe está situado en el NE de la costa cantábrica (UTM 30TWN914852). Se localiza en la cuenca del río Añarbe a 160 msnm (Unidad Hidrológica del Urumea, Cuenca Intercomunitaria Norte), entre el Territorio Histórico de Gipuzkoa (C.A.P.V.) y la Comunidad Foral de Navarra.

La cuenca vertiente del embalse se caracteriza por su relieve abrupto, con arroyos encajados en profundos valles, sin apenas llanuras de inundación y con carácter torrencial (Basoinsa, 1994). Se localiza en una zona de climatología templada-oceánica, hiperhúmeda, con temperaturas suaves y una amplitud térmica pequeña (Larumbe, 1991). En la cuenca predominan los sustratos silíceos, con alternancia de pizarras y grauvacas, que favorecen la impermeabilidad del terreno. Hoy en día, es una zona de baja actividad antrópica, aunque en los siglos pasados la explotación forestal, carboneo y minería deterioraron notablemente los bosques. La red hidrológica de la cuenca es compleja con numerosos arroyos y conducciones o derivaciones tanto para abastecimiento de la comarca de Donostia como para la producción de electricidad en centrales como las de Berdabio, Okilegi y Añarbe (Basoinsa, 1994).

El embalse se construyó en la década de 1970 y tiene como principal uso el suministro de agua potable a una población mancomunada de más de 350000 habitantes.

El área de estudio corresponde al propio embalse del Añarbe, donde se han muestreado trimestralmente tres estaciones a lo largo de su eje lon- 


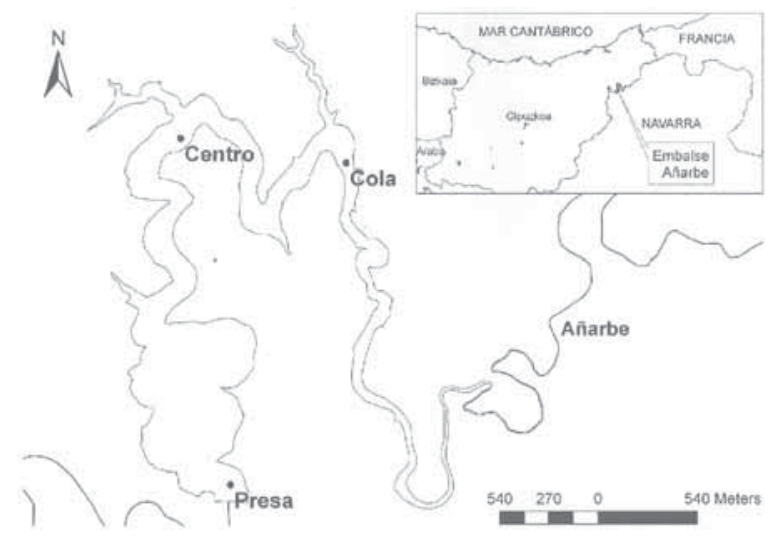

Figura 1. Área de estudio. Study area.

gitudinal (presa WN9147285302, centro y cola). Su localización aparece reflejada en la figura 1.

\section{MATERIALES Y MÉTODOS}

Se ha visitado el embalse del Añarbe con una frecuencia trimestral desde mayo de 2004 hasta enero de 2006, realizando perfiles verticales de $T^{o}$, oxígeno, $\mathrm{pH}$ y conductividad (con una sonda de profundidad HIDROLAB) en tres estaciones de su eje longitudinal (Fig. 1). La transparencia se ha estimado mediante la profundidad de visión del disco de Secchi y se han recogido muestras de agua a tres profundidades (mediante botella oceanográfica Van Dorn) para las determinaciones analíticas de nutrientes generales y metales, pigmentos y microbiología (APHA, 1992). En la zona de la presa también se han recogido muestras de fitoplancton en primavera y verano de 2004, y primavera, verano y otoño de 2005 (preservadas con Lugol y cuantificadas de acuerdo con la técnica de Utermöhl (1958).

En el año 2005 se incluyó el estudio del zooplancton en la zona de la presa (en primavera, verano y otoño), y del bentos profundo del embalse en sus tres estaciones del eje longitudinal (en primavera y otoño). El zooplancton se capturó con redes de 200 y $80 \mu \mathrm{m}$ para diferenciar mesozooplancton y microzooplancton. Se realizó un barrido vertical desde 30 metros de profundidad (muestra cualitativa) y se fijó con formol al $4 \%$ hasta su identificación. Para la recogida de las muestras de bentos profundo se ha seguido la Norma ISO 9391:1993 (E) mediante una única extracción por punto con una draga modelo Petite Ponar Grab de $0.023 \mathrm{~m}^{2}$ de superficie de muestreo.

Los datos morfométricos e hidrológicos del embalse han sido facilitados por AGASA.

\section{Estado trófico}

La concentración de fósforo total en un embalse es un parámetro crucial en la eutrofización, ya que suele ser el elemento que limita el crecimiento de las algas. La biomasa algal es un indicador de respuesta trófica y se suelen utilizar dos parámetros como estimadores. Uno es la densidad celular ( $\mathrm{n}^{\mathrm{o}}$ células $\left./ \mathrm{ml}\right)$ y el otro es la concentración de clorofila a $(\mu \mathrm{g} / \mathrm{l})$ en la zona fótica, tanto valores medios como máximos anuales. Otro parámetro relacionado con la biomasa algal, es la transparencia medida como la profundidad a la que deja de verse el disco de Secchi (valores medios y mínimos anuales).

Existen numerosos índices para estimar el estado trófico de lagos y embalses; los de más amplia utilización y por lo tanto, los más contrastados son los siguientes: OCDE (1982); Margalef (1983) y EPA (1976).

Otro método para evaluar el estado trófico es el índice de Carlson (1974) que utiliza como variables los valores medios anuales de la profundidad de visión del disco de Secchi, la concentración superficial media anual de fósforo total y de clorofila $a$. Este índice es un valor que puede variar entre oligotrofia $(<35)$; mesotrofia $(35-55)$; eutrofia (55-70) e hipereutrofia $(>70)$.

\section{Potencial ecológico}

El término potencial ecológico expresa la calidad de la estructura y funcionamiento de los ecosistemas acuáticos asociados a una masa de agua artificial o muy modificada. Dado que este tipo de masas de agua presentan en su esencia una modificación importante, hablamos de 'máximo potencial ecológico' (MPE) para designar la mejor situación de calidad posible. Las condiciones de 
referencia o MPE de estas masas de agua muy modificadas deben de aproximarse a las condiciones inalteradas de la categoría de masa de agua superficial más estrechamente comparable. En el caso de un embalse esta masa de agua es un lago. Dado que estamos hablando de masas de agua modificadas por un uso humano, las alteraciones derivadas de este uso no pueden ser obviadas o eliminadas. Así, el abastecimiento de agua potable, regadío o cualquier otro aprovechamiento del agua retenida en un embalse, provoca una oscilación en el volumen almacenado que, dentro de unos límites, consideramos algo inevitable.

La variación del volumen del embalse es el principal parámetro hidromorfológico que puede condicionar la calidad del agua embalsada. En este trabajo proponemos que para una zona con un régimen de lluvias importantes y dentro de la tipología correspondiente al embalse del Añarbe, una variación del volumen mensual de hasta el $20 \%$ del máximo puede ser acorde con el MPE para las características hidromorfológicas. Para este embalse una pérdida del $20 \%$ del volumen máximo almacenado supone un descenso de nivel de 8 metros, respecto a su cota máxima. Si el porcentaje mensual máximo de variación supera el $20 \%$, no se alcanzará el MPE. Sin embargo, analizando los elementos de calidad biológicos y fisicoquímicos podremos definir provisionalmente la clasificación del potencial ecológico como bueno, moderado, deficiente o malo (WFD CIS No 13, 2005).

El grado de eutrofización que se corresponda con el MPE también dependerá de su tipología. Así, dentro de la tipología donde se enmarca el embalse del Añarbe, proponemos que un estado oligotrófico se corresponda con un buen potencial ecológico; a un sistema mesotrófico le corresponderá un potencial ecológico moderado; a uno eutrófico, un potencial ecológico deficiente y a un medio hipereutrófico, un potencial ecológico malo.

Hasta la fecha aún no existe una metodología clara y consensuada para el establecimiento del potencial ecológico. Se han realizado importantes trabajos de aproximación intentando seguir las directrices de la DMA, tanto por parte de la Confederación Hidrográfica del Ebro (Infraestructura \& Ecología, 2003; 2006) o la Agencia
Catalana del Agua (2003; 2006), como por parte del Gobierno Vasco (2002).

En este documento se realiza una aproximación al cálculo del potencial ecológico acorde con los rangos y criterios seguidos en el documento de la Agencia Catalana del Agua (2006) y de la Confederación Hidrográfica del Ebro (Infraestructura \& Ecología, 2006), y basados en los indicadores que se han considerado en el estudio del embalse del Añarbe (Tabla 1). Para esta tipología de embalse, los valores de los distintos parámetros que separan las condiciones de oligotrófia de la mesotrófia se han considerado como el límite entre las condiciones buenas y moderadas. Así, una media anual de densidad algal total superior a $2000 \mathrm{cel} / \mathrm{ml}$ indicaría condiciones moderadas y más de $15000 \mathrm{cel} / \mathrm{ml}$, deficientes o malas (EPA, 1976). Para valorar la media y el máximo anual de clorofila a $(\mu \mathrm{g} / \mathrm{l})$ de la zona fótica, así como la media y el mínimo anual de la profundidad de visión del disco de Secchi (m) y la media anual de la concentración de fósforo total $(\mu \mathrm{gP} / \mathrm{l})$ se utilizan los rangos propuestos por la OCDE (1982). La Organización Mundial de la Salud (Chorus \& Bartram, 1999) señala $10^{5} \mathrm{cel} / \mathrm{ml}$ como la cantidad de cianobacterias con moderadas probabilidades de efectos adversos para la salud, por lo que proponemos este valor como el límite entre las condiciones deficientes y malas; y $2000 \mathrm{cel} / \mathrm{ml}$ de cianobacterias (valores máximos anuales), como el límite entre el bueno y moderado (también utilizado por la CHE, Infraestructura \& Ecología, 2006). Los valores propuestos por JRC (1992) se utilizan para valorar las condiciones de oxigenación del embalse. Así, una concentración media de oxígeno hipolimnético durante el periodo de estratificación de $6 \mathrm{mg} \mathrm{O}_{2} / \mathrm{l}$ es el límite entre el bueno y moderado; siendo el límite entre moderado y deficiente, $4 \mathrm{mg} \mathrm{O}_{2} / \mathrm{l} \mathrm{y}$ $2 \mathrm{mg} \mathrm{O}_{2} / 1$, entre deficiente y malo.

En primer lugar se realiza una valoración de cada parámetro por separado, puntuando de manera sencilla la categoría óptima con un 5 , la buena con un 4 , moderada con un 3 , deficiente con un 2 y mala con un 1. La media aritmética de los distintos parámetros de un mismo elemento nos dará la valoración de cada elemen- 
Tabla 1. Indicadores y valoración de los parámetros considerados para estimar el potencial ecológico. Propuesta por Anbiotek S.L. hasta que se aprueben unos rangos definitivos. Indicators and appraisal of the parameters considered to estimate the ecological potential. Proposal of Anbiotek until some final ranks are approved.

\begin{tabular}{|c|c|c|c|c|c|c|c|}
\hline & & \multirow[b]{3}{*}{ Puntuación } & \multicolumn{5}{|c|}{ Valoración de los parámetros } \\
\hline & & & Óptimo & Bueno & Moderado & Deficiente & Malo \\
\hline & & & 5 & 4 & 3 & 2 & 1 \\
\hline Indicador & Parámetro & & & & & & \\
\hline Biológico & & Ref. & & & & & \\
\hline \multicolumn{8}{|c|}{ Fitoplancton } \\
\hline \multicolumn{2}{|c|}{$\begin{array}{l}\text { Densidad algal total, } \\
\text { media anual }(\mathrm{cel} / \mathrm{ml})\end{array}$} & EPA, 1976 & $<1000$ & $1000-2000$ & $2000-15000$ & $>15000$ & $>15000$ \\
\hline \multicolumn{2}{|c|}{ Chl a, media anual fótica $(\mu \mathrm{g} / \mathrm{l})$} & OCDE, 1982 & $<1$ & $1-2.5$ & $2.5-8$ & $8-25$ & $>25$ \\
\hline \multicolumn{2}{|c|}{ Chl a máxima anual $(\mu \mathrm{g} / \mathrm{l})$} & OCDE, 1982 & $<2.5$ & $2.5-8$ & $8-25$ & $25-75$ & $>75$ \\
\hline \multicolumn{2}{|c|}{$\begin{array}{l}\text { Cianobacterias, máximo } \\
\text { anual }(\mathrm{cel} / \mathrm{ml})\end{array}$} & $\begin{array}{l}\text { Chorus \& Bartram } \\
\text { (1999) }\end{array}$ & $<500$ & $500-2 \cdot 10^{3}$ & $2 \cdot 10^{3}-2 \cdot 10^{4}$ & $2 \cdot 10^{4}-10^{5}$ & $>10^{5}$ \\
\hline \multicolumn{8}{|l|}{ Fisico-químico } \\
\hline \multicolumn{8}{|c|}{ Transparencia } \\
\hline \multicolumn{2}{|c|}{ Secchi, media anual (m) } & OCDE, 1982 & $>12$ & $12-6$ & $6-3$ & $3-1.5$ & $<1.5$ \\
\hline \multicolumn{2}{|c|}{ Secchi, mínimo anual (m) } & OCDE, 1982 & $>6$ & $6-3$ & $3-1.5$ & $1.5-0.7$ & $<0.7$ \\
\hline \multicolumn{8}{|c|}{ Oxigenación } \\
\hline \multicolumn{2}{|c|}{$\begin{array}{c}\text { Oxígeno hipolimnético medio durante } \\
\text { estratificación }(\mathrm{mg} / \mathrm{l})\end{array}$} & JRC, 1992 & $>8$ & $8-6$ & $6-4$ & $4-2$ & $<2$ \\
\hline \multicolumn{8}{|c|}{ Nutrientes } \\
\hline \multicolumn{2}{|c|}{ Fósforo total, media anual ( $\mu \mathrm{g} \mathrm{P} / \mathrm{l})$} & OCDE, 1982 & $<4$ & $4-10$ & $10-35$ & $35-100$ & $>100$ \\
\hline \multicolumn{2}{|c|}{$\begin{array}{c}\text { APROXIMACIÓN AL POTENCIAL } \\
\text { ECOLÓGICO }\end{array}$} & \multicolumn{6}{|c|}{$\begin{array}{l}\text { La valoración del peor de los indicadores } \\
\text { (biológico o fisicoquímico) }\end{array}$} \\
\hline
\end{tabular}

to considerado. Así hay elementos (E) compuestos por un solo parámetro $(\mathrm{P})$ como Enutrientes (= P fósforo total anual) o Eoxigenación (= P oxígeno hipolimnético) y otros, compuestos por dos o más, como Efitoplancton (PChl media, PChl máx, PDensidad algal, PCianofíceas cel/ml), o Etransparencia (Psecchi medio, Psecchi min).

Siguiendo la metodología descrita por la Agencia Catalana del Agua (2003) para obtener el valor de los indicadores fisicoquímicos se calcula la media de sus elementos; en cambio el valor de los indicadores biológicos se obtendrá considerando la valoración del peor de sus elementos. Finalmente el valor provisional del potencial ecológico se obtendrá del indicador (biológico o fisicoquímico) con una peor valoración ecológica.

La Agencia Catalana del Agua cuenta con masas de agua de referencia para cada tipo de embalse, por lo que pueden estimar el grado de des- viación del potencial ecológico máximo. Sin embargo, en la Cuenca Norte no hay establecidas condiciones de referencia para embalses. Por ello la clasificación provisional del potencial ecológico para los embalses estará representada por el peor de los valores de los indicadores (biológicos o fisicoquímicos).

\section{RESULTADOS Y DISCUSIÓN}

\section{Caracterización y tipificación del embalse del Añarbe}

El embalse del Añarbe se localiza en la Región ibérico-macaronésica y ha sido designado provisionalmente como 'masa de agua muy modificada' asimilable a 'lago'. Según el Sistema A de clasificación de la DMA se corresponde a un tipo 'silíceo, tierras bajas, grande y muy profundo'; 

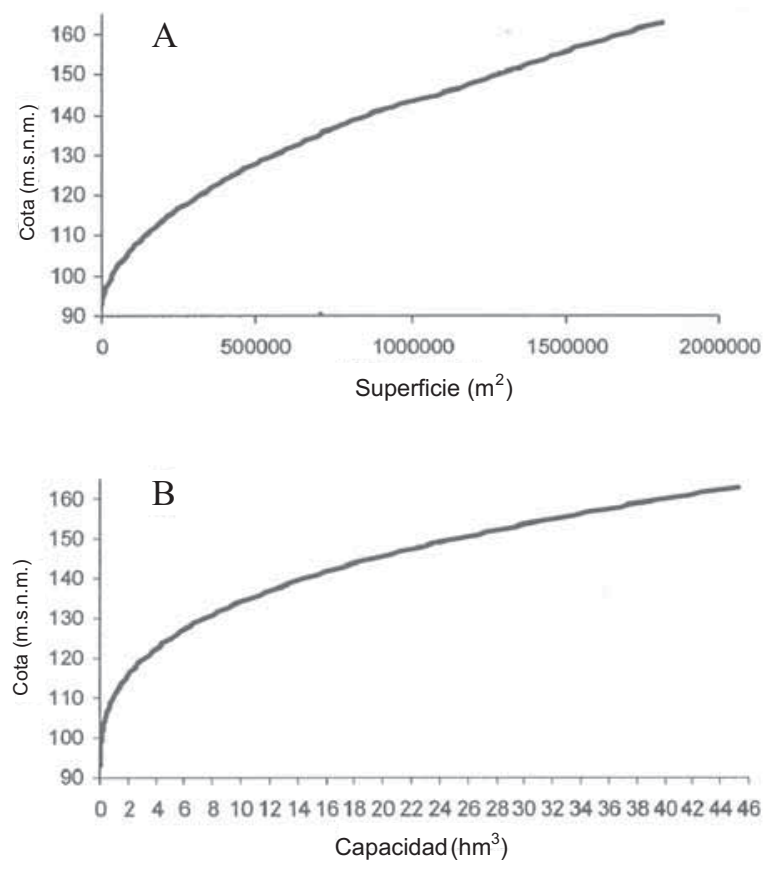

Figura 2. A.- Curva hipsográfica (cota-superficie). B.- Relación cota-capacidad en el embalse del Añarbe. Fuente: AGASA. A.- Hipsographic curve(level-surface). B.- level-capacity relation for the Añarbe reservoir. Source: AGASA.

y en base al Sistema B correspondería a un tipo 'tierras bajas, septentrional, monomíctico cálido y de aguas ácidas'.

Hasta la fecha no hay establecida ninguna masa de agua de referencia con estas características en la Cuenca Norte.

La masa de agua natural más similar al embalse del Añarbe en la Península es la lago de Sanabria (Zamora), clasificado como 'silíceo, alto, grande y muy profundo' según el Sistema A. En cuanto al Sistema B, el lago de Sanabria es 'de media montaña, monomíctico cálido y de aguas ácidas' . La diferencia es la altitud a la que se encuentran ambas masas de agua: el embalse se localiza a menos de $200 \mathrm{~m}$ de altitud y el lago a más de $800 \mathrm{~m}$.

\section{Indicadores hidromorfológicos}

La morfometría de un embalse y la importancia relativa de su cuenca vertiente determinan en gran medida sus características fisicoquímicas y biológicas (Hutchinson, 1957; Wetzel, 1981; Häkanson, 1981; Catalán, 1987).
Tabla 2. Parámetros morfométricos del embalse de Añarbe. Morphometric parameters of the Añarbe reservoir.

\begin{tabular}{lc}
\hline \multicolumn{2}{c}{ PARÁMETROS MORFOMÉTRICOS } \\
\hline Superficie de la cuenca total (ha) & 6900 \\
Superficie del embalse (ha) & 201 \\
\% Cuenca ocupado por embalse Añarbe & $2.91 \%$ \\
Longitud total del embalse (m) & 7400 \\
Anchura máxima (m) & 674 \\
Profundidad máxima (m) & 65 \\
Profundidad media (m) & 21.74 \\
Perímetro en nivel máximo (m) (pista perimetral) & 20000 \\
Cota a máximo nivel embalsado (msnm) & 160 \\
Cota(s) de la toma(s) de agua principal(es) (msnm) & 122 y 110 \\
Capacidad máxima (hm ${ }^{3}$ ) & 43.7 \\
Capacidad útil (hm ${ }^{3}$ ) & 37.3 \\
Profundidad relativa (Zr) \% & 4.06 \\
Zm:Zmax & 0.33 \\
Desarrollo de volumen (Dv) & 1.00 \\
Desarrollo del litoral (DL) & 3.98 \\
Ac/V m ${ }^{-1}$ & 1.58 \\
Ac/A & 34.33 \\
\hline
\end{tabular}

La variación del área y volumen, con relación a la profundidad se puede observar en la figura 2 . Los parámetros e índices del embalse del Añarbe (Tabla 2) señalan que la cubeta tiene una forma asimilable a un cono invertido con una gran pendiente en sus márgenes (relación Zm:Zmax igual a 0.33 y Dv de 1) y una profundidad relativa que favorece la estabilidad durante la estratificación térmica ( $\mathrm{Zr}$ de $4.06 \%$ ). Su forma es sinuosa con gran superficie de contacto entre el embalse y el medio terrestre circundante (DL igual a 3.98). La relación entre la cuenca vertiente y el volumen o el área del embalse (Ac/V y Ac/A) es bastante elevada e indica que la masa de agua es sensible a presentar problemas de eutrofia, debido a la gran repercusión que cualquier modificación en los usos del suelo de la cuenca pueden producir sobre el estado trófico del embalse.

El volumen máximo del embalse se alcanza en invierno y el mínimo en el mes de noviembre (Fig. 3). Las aportaciones máximas al embalse por el río Añarbe y sus afluentes se producen entre diciembre y febrero, coincidiendo con la época de lluvias más importantes; además hay otro pico importante con el deshielo de primavera en abril. El caudal de salida mayor se produce en febrero y en abril; por otra parte, el uso para abas- 
A
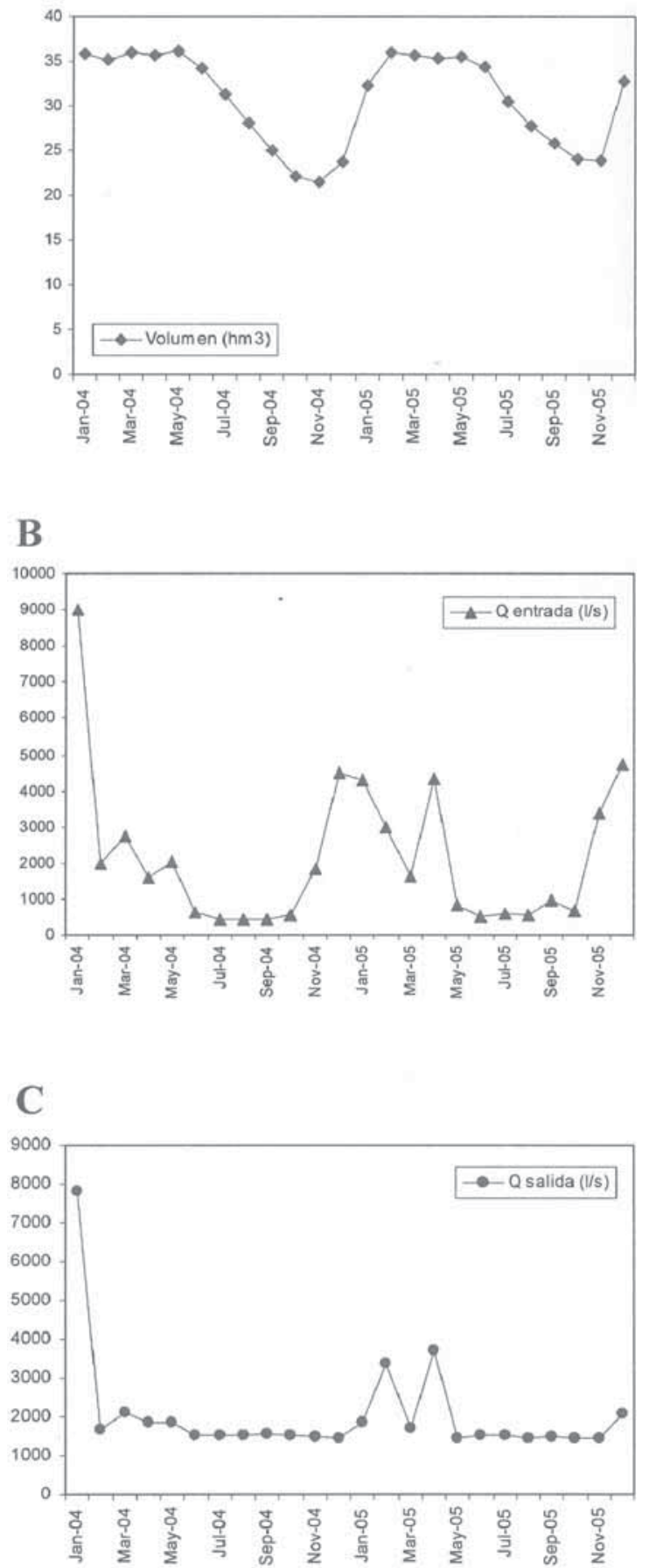

Figura 3. A.- Variación del volumen embalsado $\left(\mathrm{hm}^{3}\right)$ en el embalse del Añarbe (enero 2004-diciembre 2005). B.- Caudal de entrada (Q, 1/s). C.- Caudal de salida mensual (Q, 1/s). A.Variation of the dammed volume $\left(\mathrm{hm}^{3}\right)$ in the Añarbe reservoir (January 2004-December 2005). B.- Entry volume (Q, l/s). C.Monthly exit volume $(Q, l / s)$.
Tabla 3. Principales parámetros hidráulicos del embalse del Añarbe. Main hydraulic parameters of the Añarbe reservoir.

\begin{tabular}{|c|c|c|}
\hline \multicolumn{3}{|c|}{ PARÁMETROS HIDRÁULICOS } \\
\hline & Año 2004 & Año 2005 \\
\hline Volumen medio $\left(\mathrm{hm}^{3}\right)$ & 30.39 & 31.12 \\
\hline Superficie media (ha) & 142.35 & 143.53 \\
\hline Profundidad media (m) & 21.34 & 21.68 \\
\hline Entradas $\left(\mathrm{hm}^{3} / \mathrm{año}\right)$ & 69.31 & 66.79 \\
\hline Salidas (hm³/año) & 68.33 & 60.09 \\
\hline Tiempo de retención hidráulico Tw (años) & 0.44 & 0.46 \\
\hline Tasa de renovación D (años $\left.{ }^{-1}\right)$ & 2.3 & 2.14 \\
\hline
\end{tabular}

tecimiento de este embalse cuenta con una concesión de 1500 l/s que es la salida, más o menos constante, en el resto de los meses.

El tiempo de retención hidráulico determina el tiempo de que dispone un determinado proceso para llevarse a cabo en el embalse (por ejemplo, el crecimiento del plancton). Los tiempos de residencia hidráulica de los embalses españoles oscilan entre 0.5 y 50 años (Margalef, 1983) y el embalse del Añarbe se encuentra entre los que tienen un menor tiempo de residencia (Tw de 0.4 años), lo que implica que se renueva algo más de 2 veces en un año (Tabla 3). El porcentaje de variación del volumen con respecto al volumen máximo es mayor en el mes de noviembre, siendo del $40 \%$ en 2004 y del $33 \%$ en 2005 (Tabla 4).

Tabla 4. Porcentaje de variación del volumen con respecto al volumen máximo en el embalse del Añarbe. Volume variation percentage respective to the maximum volume in the Añarbe reservoir.

\begin{tabular}{lcc}
\hline \multicolumn{1}{c}{ Meses } & $\begin{array}{c}\text { \% Variación } \\
\text { volumen 2004 }\end{array}$ & $\begin{array}{c}\text { \% Variación } \\
\text { volumen 2005 }\end{array}$ \\
\hline Enero & 1.11 & 9.97 \\
Febrero & 2.65 & 0.00 \\
Marzo & 0.50 & 0.92 \\
Abril & 1.33 & 1.56 \\
Mayo & 0.00 & 1.06 \\
Junio & 5.31 & 4.40 \\
Julio & 13.60 & 15.29 \\
Agosto & 22.25 & 22.89 \\
Septiembre & 31.01 & 28.35 \\
Octubre & 38.89 & 33.25 \\
Noviembre & 40.80 & 33.36 \\
Diciembre & 34.66 & 8.99 \\
\hline Variación & & \\
máxima anual & $\mathbf{4 0 \%}$ & $\mathbf{3 3 . 3 \%}$ \\
\hline
\end{tabular}



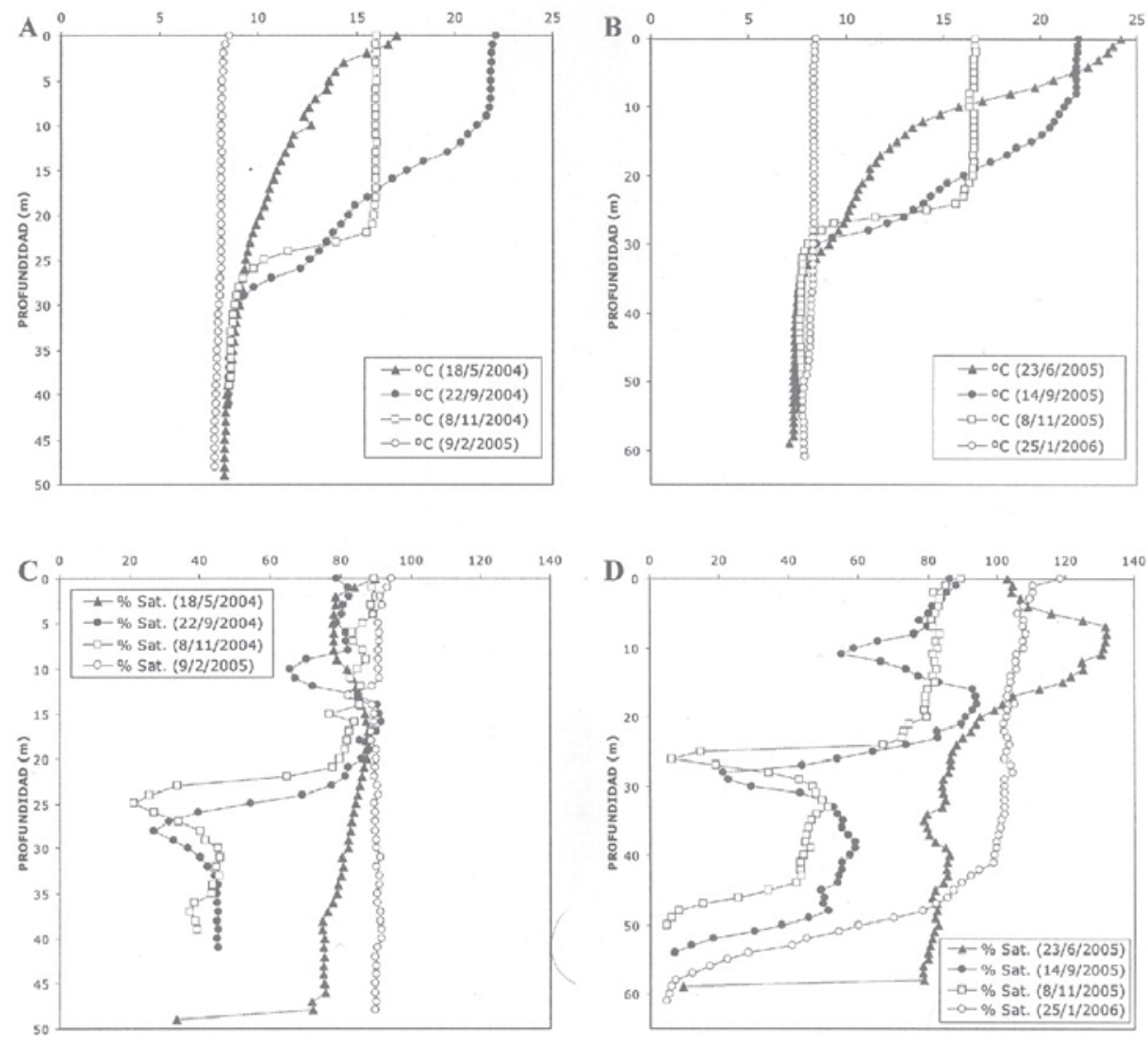

Figura 4. Perfiles verticales de temperatura (A y B) y \% saturación de oxígeno (C y D) en la presa del embalse del Añarbe. Vertical temperature profiles $(A$ and $B)$ and percentage of oxygen saturation $(C$ and $D)$ in the dam of the Añarbe reservoir.

\section{Indicadores fisicoquímicos}

El embalse del Añarbe se clasifica como monomíctico cálido y presenta en la zona más profunda de la presa una estratificación térmica que comienza en primavera y finaliza en diciembre (Fig. 4). La termoclina estacional se localiza en verano entre los 25 y $30 \mathrm{~m}$ de profundidad, existiendo también una termoclina secundaria en el epilimnion entre los 10 y $15 \mathrm{~m}$, que se forma debido al intenso calentamiento superficial resultado del ciclo nictemeral en verano (Fig. 4).

El embalse del Añarbe no presenta problemas graves de anoxia en el periodo 2004/2006. Sin embargo, en la presa y en el centro del embalse en verano se observa un mínimo relativo de oxígeno asociado a la termoclina (tanto estacional como secundaria); y en otoño e incluso en invierno, estos mínimos de oxígeno se mantie- nen en la presa, asociados a la cubeta profunda en la que la mezcla aún no se ha completado (Fig. 4). Hay que señalar que los mínimos relativos de oxígeno son más acusados en el periodo 2005/2006 que en el periodo anual 2004/2005. La termoclina actúa como una 'barrera' sobre la que tienden a acumularse microorganismos, materia orgánica e inorgánica disuelta y sales minerales que contribuyen al aumento relativo de la conductividad (Fig. 5) y a la disminución relativa de oxígeno. La concentración media de oxígeno hipolimnético durante el periodo de estratificación es de $4.5 \mathrm{mg} \mathrm{O}_{2} / 1$.

El embalse del Añarbe tiene su máxima transparencia en la zona de la presa, donde la capa fótica media alcanza entre los 17 y los $19 \mathrm{~m}$ de profundidad. Los valores medios y mínimos anuales de la profundidad de visión del disco de Secchi en la zona de la presa indican condiciones 

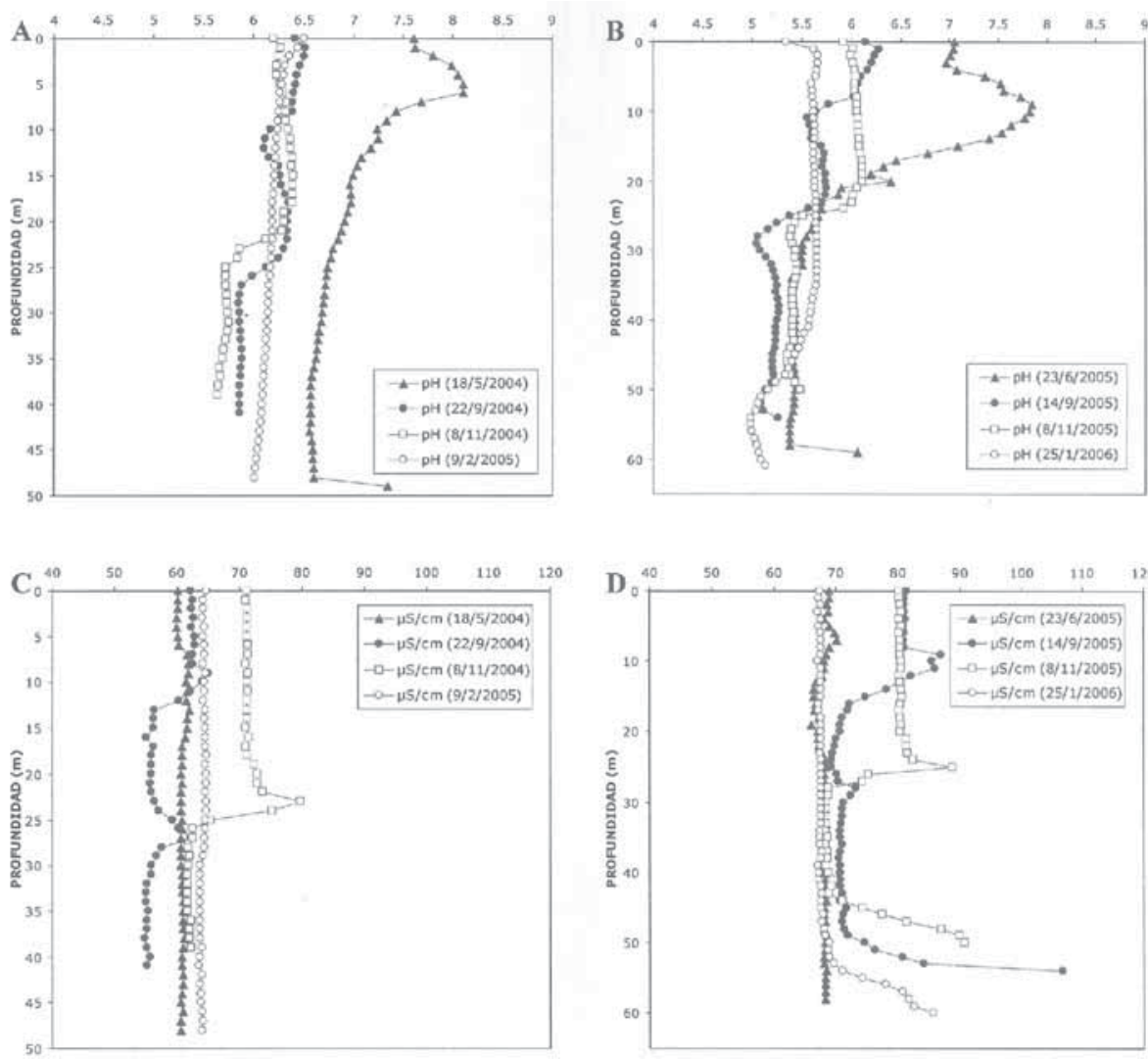

Figura 5. Perfiles verticales de $\mathrm{pH}(\mathrm{A}$ y B $)$ y conductividad $(\mu \mathrm{S} / \mathrm{cm})(\mathrm{C}$ y D) en la presa del embalse del Añarbe. Vertical $p H(A$ and $B)$ and conductivity $(\mu \mathrm{S} / \mathrm{cm})(C$ y $D)$ profiles in the dam of the Añarbe reservoir.

de oligotrofia. La transparencia disminuye progresivamente hacia la cola, como corresponde al carácter más fluvial de esta zona.

Las aguas del embalse son ácidas, con los valores de $\mathrm{pH}$ más bajos en la presa, sobre todo en verano y en el hipolimnion (Fig. 5). Tienen una mineralización débil y los valores medios anuales indican una composición iónica bicarbonatada clorurada cálcico sódica (Tabla 5).

De las forma del nitrógeno, el nitrato es la más abundante, pero en concentraciones muy bajas (entre 1.5 y $3.5 \mathrm{mg} \mathrm{NO}_{3} / \mathrm{l}$ ). La peor calidad se da en la presa (en el periodo 2005/2006) cuando se obtiene una calidad tipo A2 por la concentración de amonio y una calidad tipo ciprinícola para la vida piscícola según los niveles de nitrito (R.D. 927/1988, de 29 de julio).

Las concentraciones de fósforo en el embalse son inferiores en el ciclo anual 2005/2006, que en el anterior (Tabla 5); sin embargo en ambos periodos se encuentran dentro del rango de la mesotrofia. El aluminio y el sílice presentan concentraciones muy homogéneas en el embalse.

Los metales hierro y manganeso presentan las concentraciones puntuales más elevadas cuando el embalse está en su cota más baja, esto es en verano y otoño. El hierro no presenta problemas para la calidad de las aguas; si bien, el manganeso tiene una concentración media en la presa que implica un nivel de tratamiento de tipo A3 para producción de agua potable (R.D. 927/1988, de 29 de julio). Los niveles de manganeso en el sustrato pizarroso y granítico de la cuenca del Añarbe son elevados (Basoinsa, 1994) y su movilidad hacia la fase líquida, dado el $\mathrm{pH}$ ácido de las aguas, es también alto.

El contenido de materia orgánica, determinado a partir de la $\mathrm{DBO}_{5}$ y DQO es bajo y no pre- 
Tabla 5. Valores medios de las principales variables fisicoquímicas y de la clorofila a para cada estación de muestreo del embalse (presa, centro y cola) desde mayo de 2004 a febrero de 2005 (2004/2005) y desde junio de 2005 a enero de 2006 (2005/2006). Entre paréntesis se señala la desviación estándar (DS) y el mínimo y máximo (min-max) de cada variable. Average values of the main physicochemical variables and of the chlorophyll-a for each sampling station of the reservoir (dam, centre and tail) from May of 2004 to February of 2005 (2004/2005) and from June of 2005 to January of 2006 (2005/2006). Standard deviation (SD) and minimum and maximum (min-max) for each variable are shown between brackets.

\begin{tabular}{|c|c|c|c|c|c|c|}
\hline \multirow[b]{2}{*}{ Periodo } & \multicolumn{2}{|c|}{ PRESA } & \multicolumn{2}{|c|}{ CENTRO } & \multicolumn{2}{|c|}{ COLA } \\
\hline & 2004-05 & 2005-06 & 2004-05 & 2005-06 & 2004-05 & 2005-06 \\
\hline Prof. Secchi (m) & $\begin{array}{l}7.40(2.6) \\
(4.9-10.5)\end{array}$ & $\begin{array}{c}8.50(2.6) \\
(5.80-11.00)\end{array}$ & $\begin{array}{c}6.28(1.7) \\
(4.60-8.50)\end{array}$ & $\begin{array}{c}6.90(1.1) \\
(5.5-8.1)\end{array}$ & $\begin{array}{c}5.70(2.3) \\
(4.40-9.10)\end{array}$ & $\begin{array}{c}5.78(1.4) \\
(4.50-7.50)\end{array}$ \\
\hline Chl a $(\mu \mathrm{g} / \mathrm{l})$ & $\begin{array}{c}0.76(0.7) \\
(0.06-2.36)\end{array}$ & $\begin{array}{c}0.27(0.2) \\
(0.01-0.55)\end{array}$ & $\begin{array}{c}1.10(1.4) \\
(0.15-4.46)\end{array}$ & $\begin{array}{c}0.84(0.9) \\
(0.04-2.04)\end{array}$ & $\begin{array}{l}2.25(2.4) \\
(0.41-8.7)\end{array}$ & $\begin{array}{c}0.49(0.5) \\
(0-1.25)\end{array}$ \\
\hline $\mathrm{Ca}^{++}(\mathrm{meq} / \mathrm{l})$ & $\begin{array}{c}0.41(0.1) \\
(0.31-0.64)\end{array}$ & $\begin{array}{l}0.36(0.08) \\
(0.29-0.52)\end{array}$ & $\begin{array}{l}0.37(0.05) \\
(0.28-0.44)\end{array}$ & $\begin{array}{l}0.36(0.05) \\
(0.30-0.42)\end{array}$ & $\begin{array}{l}0.39(0.07) \\
(0.30-0.50)\end{array}$ & $\begin{array}{l}0.37(0.05) \\
(0.31-0.45)\end{array}$ \\
\hline $\mathrm{Mg}^{++}(\mathrm{meq} / \mathrm{l})$ & $\begin{array}{l}0.17(0.13) \\
(0.05-0.42)\end{array}$ & $\begin{array}{l}0.09(0.01) \\
(0.06-0.10)\end{array}$ & $\begin{array}{c}0.18(0.2) \\
(0.01-0.51)\end{array}$ & $\begin{array}{l}0.09(0.01) \\
(0.07-0.11)\end{array}$ & $\begin{array}{l}0.13(0.08) \\
(0.08-0.37)\end{array}$ & $\begin{array}{l}0.09(0.01) \\
(0.07-0.11)\end{array}$ \\
\hline $\mathrm{Na}^{+}(\mathrm{meq} / \mathrm{l})$ & $\begin{array}{l}0.29(0.07) \\
(0.24-0.48)\end{array}$ & $\begin{array}{l}0.23(0.02) \\
(0.21-0.26)\end{array}$ & $\begin{array}{l}0.29(0.05) \\
(0.23-0.35)\end{array}$ & $\begin{array}{l}0.23(0.02) \\
(0.21-0.26)\end{array}$ & $\begin{array}{l}0.28(0.05) \\
(0.22-0.35)\end{array}$ & $\begin{array}{l}0.23(0.02) \\
(0.21-0.26)\end{array}$ \\
\hline $\mathrm{K}^{+}(\mathrm{meq} / \mathrm{l})$ & $\begin{array}{l}0.03(0.01) \\
(0.03-0.04)\end{array}$ & $\begin{array}{l}0.02(0.00) \\
(0.01-0.03)\end{array}$ & $\begin{array}{l}0.03(0.01) \\
(0.03-0.05)\end{array}$ & $\begin{array}{l}0.02(0.00) \\
(0.01-0.03)\end{array}$ & $\begin{array}{l}0.03(0.00) \\
(0.02-0.03)\end{array}$ & $\begin{array}{l}0.02(0.00) \\
(0.01-0.03)\end{array}$ \\
\hline $\mathrm{HCO}_{3}^{-}(\mathrm{meq} / \mathrm{l})$ & $\begin{array}{l}0.39(0.11) \\
(0.27-0.59)\end{array}$ & $\begin{array}{l}0.42(0.09) \\
(0.33-0.64)\end{array}$ & $\begin{array}{l}0.38(0.08) \\
(0.27-0.50)\end{array}$ & $\begin{array}{l}0.41(0.05) \\
(0.32-0.48)\end{array}$ & $\begin{array}{l}0.40(0.08) \\
(0.30-0.51)\end{array}$ & $\begin{array}{l}0.43(0.04) \\
(0.36-0.49)\end{array}$ \\
\hline $\mathrm{SO}_{4}^{-}(\mathrm{meq} / \mathrm{l})$ & $\begin{array}{l}0.12(0.04) \\
(0.07-0.17)\end{array}$ & $\begin{array}{l}0.09(0.05) \\
(0.05-0.21)\end{array}$ & $\begin{array}{l}0.13(0.05) \\
(0.09-0.19)\end{array}$ & $\begin{array}{l}0.10(0.06) \\
(0.06-0.23)\end{array}$ & $\begin{array}{l}0.11(0.03) \\
(0.07-0.16)\end{array}$ & $\begin{array}{l}0.10(0.05) \\
(0.06-0.21)\end{array}$ \\
\hline $\mathrm{Cl}^{-}(\mathrm{meq} / \mathrm{l})$ & $\begin{array}{l}0.14(0.05) \\
(0.08-0.20)\end{array}$ & $\begin{array}{l}0.18(0.02) \\
(0.17-0.22)\end{array}$ & $\begin{array}{l}0.14(0.04) \\
(0.08-0.18)\end{array}$ & $\begin{array}{l}0.18(0.02) \\
(0.14-0.21)\end{array}$ & $\begin{array}{l}0.13(0.03) \\
(0.10-0.16)\end{array}$ & $\begin{array}{l}0.19(0.02) \\
(0.17-0.21)\end{array}$ \\
\hline $\mathrm{NH}_{4}^{+}(\mathrm{mg} / \mathrm{l})$ & $\begin{array}{c}0.03(0.03) \\
(0-0.09)\end{array}$ & $\begin{array}{l}0.11(0.16) \\
(0-0.38)\end{array}$ & $\begin{array}{c}0.04(0.04) \\
(0-0.09)\end{array}$ & $\begin{array}{l}0.01(0.01) \\
(0-0.02)\end{array}$ & $\begin{array}{c}0.04(0.04) \\
(0-0.12)\end{array}$ & $\begin{array}{l}0.05(0.07) \\
(0-0.23)\end{array}$ \\
\hline $\mathrm{NO}_{2}^{-}(\mathrm{mg} / \mathrm{l})$ & $\begin{array}{c}0.01(0.01) \\
(0-0.03)\end{array}$ & $\begin{array}{c}0.03(0.05) \\
(0-0.19)\end{array}$ & $\begin{array}{c}0.01(0.01) \\
(0-0.02)\end{array}$ & $\begin{array}{l}0.02(0.01) \\
(0.01-0.03)\end{array}$ & $\begin{array}{c}0.01(0.01) \\
(0-0.03)\end{array}$ & $\begin{array}{l}0.02(0.00) \\
(0.01-0.02)\end{array}$ \\
\hline $\mathrm{NO}_{3}^{-}(\mathrm{mg} / \mathrm{l})$ & $\begin{array}{l}2.46(0.59) \\
(1.08-3.04)\end{array}$ & $\begin{array}{l}2.47(0.68) \\
(1.30-3.36)\end{array}$ & $\begin{array}{l}2.59(0.49) \\
(1.89-3.24)\end{array}$ & $\begin{array}{l}2.47(0.54) \\
(1.61-3.10)\end{array}$ & $\begin{array}{c}2.28(0.43) \\
(1.6-2.85)\end{array}$ & $\begin{array}{l}2.31(0.62) \\
(1.47-3.20)\end{array}$ \\
\hline NTK (mg/l) & $\begin{array}{l}0.40(0.5) \\
(0-1.12)\end{array}$ & $\begin{array}{c}0.17(0.33) \\
(0-1.00)\end{array}$ & $\begin{array}{c}0.28(0.35) \\
(0-0.96)\end{array}$ & $\begin{array}{c}0.25(0.58) \\
(0-2.00)\end{array}$ & $\begin{array}{c}0.86(0.96) \\
(0-3.00)\end{array}$ & $\begin{array}{c}1.38(3.21) \\
(0-10.00)\end{array}$ \\
\hline $\mathrm{P}^{-} \mathrm{PO}_{4}^{3-}(\mu \mathrm{gP} / \mathrm{l})$ & $\begin{array}{c}3.83(4.53) \\
(0-10.00)\end{array}$ & $\begin{array}{c}1.58(2.62) \\
(0-8.70)\end{array}$ & $\begin{array}{c}3.48(4.29) \\
(0-10.00)\end{array}$ & $\begin{array}{c}0.96(1.07) \\
(0-2.60)\end{array}$ & $\begin{array}{c}3.81(4.41) \\
(0-10.00)\end{array}$ & $\begin{array}{c}1.13(1.31) \\
(0-3.90)\end{array}$ \\
\hline P Total $(\mu \mathrm{gP} / \mathrm{l})$ & $\begin{array}{l}27.01(37.5) \\
(0.1-127.14)\end{array}$ & $\begin{array}{c}11.82(8.36) \\
(0-31.00)\end{array}$ & $\begin{array}{l}26.90(29.4) \\
(0.1-101.00)\end{array}$ & $\begin{array}{l}27.92(36.2) \\
(6.5-132.40)\end{array}$ & $\begin{array}{l}37.30(39.5) \\
(9.6-132.85)\end{array}$ & $\begin{array}{l}22.35(25.5) \\
(3.10-92.70)\end{array}$ \\
\hline $\mathrm{SiO}_{2}(\mathrm{mg} / \mathrm{l})$ & $\begin{array}{c}12.46(1.5) \\
(10.37-15.6)\end{array}$ & $\begin{array}{c}11.23(2.4) \\
(7.54-14.52)\end{array}$ & $\begin{array}{l}11.61(2.34) \\
(8.06-16.40)\end{array}$ & $\begin{array}{l}11.06(4.01) \\
(3.71-17.61)\end{array}$ & $\begin{array}{c}11.83(1.7) \\
(8.16-14.90)\end{array}$ & $\begin{array}{c}11.29(3.0) \\
(6.47-15.29)\end{array}$ \\
\hline Aluminio (mg/l) & $\begin{array}{l}0.01(0.01) \\
(0-0.04)\end{array}$ & $\begin{array}{l}0.02(0.01) \\
(0.01-0.05)\end{array}$ & $\begin{array}{c}0.03(0.04) \\
(0-0.12)\end{array}$ & $\begin{array}{l}0.03(0.01) \\
(0.01-0.06)\end{array}$ & $\begin{array}{l}0.02(0.02) \\
(0-0.06)\end{array}$ & $\begin{array}{l}0.03(0.01) \\
(0.01-0.05)\end{array}$ \\
\hline Fe total $(\mu \mathrm{g} / \mathrm{l})$ & $\begin{array}{c}96.58(143.4) \\
(13-531)\end{array}$ & $\begin{array}{c}51.75(39.9) \\
(17-164)\end{array}$ & $\begin{array}{c}115.83(83.8) \\
(52-362)\end{array}$ & $\begin{array}{c}167.0(165.5) \\
(39-554)\end{array}$ & $\begin{array}{c}127.9(114.1) \\
(35-433)\end{array}$ & $\begin{array}{c}111.5(75.3) \\
(35-286)\end{array}$ \\
\hline Fe disuelto $(\mu \mathrm{g} / \mathrm{l})$ & $\begin{array}{c}28.33(19.4) \\
(5-71)\end{array}$ & $\begin{array}{c}34.08(33.7) \\
(8-136)\end{array}$ & $\begin{array}{c}84.11(89.7) \\
(25-307)\end{array}$ & $\begin{array}{c}73.50(63.5) \\
(30-249)\end{array}$ & $\begin{array}{c}90.44(95.8) \\
(28-329)\end{array}$ & $\begin{array}{c}88.00(66.1) \\
(29-232)\end{array}$ \\
\hline Mn total $(\mu \mathrm{g} / \mathrm{l})$ & $\begin{array}{c}109.9(281.4) \\
(7-1000)\end{array}$ & $\begin{array}{l}153.1(403.0) \\
(6-1420)\end{array}$ & $\begin{array}{c}67.92(87.2) \\
\quad(7-258)\end{array}$ & $\begin{array}{c}52.67(102.3) \\
(6-374)\end{array}$ & $\begin{array}{c}45.42(48.1) \\
(11-173)\end{array}$ & $\begin{array}{c}79.75(152.1) \\
(6-410)\end{array}$ \\
\hline Mn disuelto $(\mu \mathrm{g} / \mathrm{l})$ & $\begin{array}{c}96.21(253.5) \\
(4-897.50)\end{array}$ & $\begin{array}{c}96.17(246.1) \\
(0.50-860)\end{array}$ & $\begin{array}{c}58.33(80.5) \\
(7-233)\end{array}$ & $\begin{array}{c}29.08(54.7) \\
(0-197)\end{array}$ & $\begin{array}{c}33.50(37.1) \\
(7-117)\end{array}$ & $\begin{array}{c}45.58(115.3) \\
(0.50-405)\end{array}$ \\
\hline
\end{tabular}


senta problemas ya que se encuentra por debajo de los límites más exigentes de calidad recomendados para producción de agua potable.

\section{Indicadores biológicos}

En los muestreos de verano, tanto de 2004 como de 2005, se observó la presencia bastante abundante de un pequeño cnidario, probablemente Craspedacus- ta sowerbii, que corresponde a medusas de agua dulce, cuya presencia ya se ha observado en otros embalses de la península (Pérez-Bote et al., 2005; Alonso, comunicación personal).

Los valores de clorofila $a$ son muy bajos, acordes con la baja densidad fitoplanctónica encontrada (inferior a $2000 \mathrm{cel} / \mathrm{ml}$ ) e indicativos de condiciones de oligotrofia (Tabla 5).

En el año 2004 se estudió el fitoplancton en

Tabla 6. Densidad (cel/ml) del fitoplancton en el epilimnion de la presa del Añarbe. Phytoplankton density (cell/ml) in the epilimnion of the dam of the Añarbe reservoir.

\begin{tabular}{|c|c|c|c|c|c|}
\hline \multirow[b]{2}{*}{ TAXONES } & \multicolumn{5}{|c|}{ PRESA DEL AÑARBE } \\
\hline & $18 / 5 / 04$ & 8/11/04 & $23 / 6 / 05$ & $14 / 9 / 05$ & $8 / 11 / 05$ \\
\hline \multicolumn{6}{|l|}{ Cianofíceas } \\
\hline Chroococcus sp. & & & & & 15.15 \\
\hline Coelosphaerium pusillum & & & & & 60.60 \\
\hline Cyanogranis sp. & & 1.00 & & & 30.30 \\
\hline Oscillatoria sp. & & 1.00 & & & 1.00 \\
\hline Synechococcus sp. & & & & & 196.95 \\
\hline \multicolumn{6}{|l|}{ Clorofíceas } \\
\hline Botryococcus braunii & & & 19 & 160.90 & \\
\hline Chlamydomonas sp. & & & 3 & 30.30 & \\
\hline Chlorella sp. & & & 2 & 30.30 & 1.00 \\
\hline Chlorolobion lunulatum & & & & & 15.15 \\
\hline Clorococal indet. & & & & 30.30 & 1.00 \\
\hline Closterium lunula & & & 1 & 15.15 & \\
\hline Coenoccocus sp. & & 30.30 & & & \\
\hline Coenochloris piscinalis & & 1.00 & & & \\
\hline Dictyosphaerium pulchellum & & & & & 121.20 \\
\hline Eutetramorus fottii & & & 173 & 145.75 & \\
\hline Hyaloraphidium curvatum & & & 1.00 & 15.15 & \\
\hline Oocystis lacustris & 60.60 & 1.00 & 7 & 15.15 & \\
\hline Pandorina morum & & & 8 & 30.30 & \\
\hline Planktosphaeria gelatinosa & 45.45 & & & & \\
\hline Radiocystis sp. & & & & 45.45 & \\
\hline Scenedesmus armatus & & & & 30.30 & \\
\hline Scenedesmus disciformis & & & 16 & & \\
\hline Scenedesmus dispar & & & 2 & 15.15 & \\
\hline Scenedesmus ellipticus & & & & & 30.30 \\
\hline \multirow{2}{*}{\multicolumn{6}{|c|}{ Crisófitos }} \\
\hline & & & & & \\
\hline \multicolumn{6}{|l|}{ Bacilariofíceas } \\
\hline Cyclotella sp. & 2.00 & 60.60 & & & \\
\hline Cyclotella cf. stelligera & & 85.75 & & & 15.15 \\
\hline Cyclotella meneghiniana & & & 3 & 45.45 & \\
\hline Fragilaria sp. & & & & 15.15 & \\
\hline Navicula sp. & & 1.00 & 1 & 15.15 & \\
\hline Nitzschia amphibia & & & & 15.15 & \\
\hline Tabellaria flocculosa & & & 2 & & \\
\hline \multicolumn{6}{|l|}{ Xantofíceas } \\
\hline Characiopsis saccata & & & & 15.15 & \\
\hline \multicolumn{6}{|l|}{ Dinofíceas } \\
\hline Peridinium sp. & 45.45 & 1.00 & 84 & 166.65 & 1.00 \\
\hline \multicolumn{6}{|l|}{ Euglenofíceas } \\
\hline Trachelomonas volvocina & 30.30 & 1.00 & & & \\
\hline Densidad (cel/ml) & 185.80 & 209.80 & 325 & 836.90 & 488.80 \\
\hline
\end{tabular}


primavera y otoño (Tabla 6). En la presa, el grupo más abundante corresponde a las clorofíceas (Oocystis lacustris) en primavera; y a las diatomeas (Cyclotella sp.) en otoño, con presencia puntual en esta campaña de cianofíceas.

En el año 2005 se estudió el fitoplancton en primavera, verano y otoño (Tabla 6). Los clorófitos y dinófitos son los principales representantes del fitoplancton en primavera; en verano, el grupo claramente dominante son las algas clorofíceas; mientras que en otoño se produce un incremento notable en la presencia de cianofíceas de pequeño tamaño (Synechococcus sp. y Coelosphaerium pusillum) que pasa a ser el grupo más abundante.

El zooplancton se ha estudiado en la zona de la presa del embalse en primavera y otoño de 2005. En general, en cada campaña de muestreo se encuentra una comunidad bien representada formada principalmente por una o dos especies de Daphnia o Ceriodaphnia; una o dos especies de copépodos en menor abundancia; y rotíferos, con mayor diversidad pero menor abundancia. Están presentes especies de aguas poco mineralizadas, como los cladóceros $\mathrm{Ce}$ riodaphnia quadrangula, Daphnia longispina,
D. pulicaria, D. galeata, Bosmina longirostris o D. cucullata, esta última poco frecuente; así como la especie Ploesoma hudsoni, rotífero encontrado también en otros embalses del NW de la Península. En los embalses, la mayor tasa de renovación frente a los lagos favorece a las especies de rápida multiplicación (rotíferos y cladóceros) frente a los copépodos.

El bentos profundo del embalse del Añarbe se ha estudiado en primavera y otoño de 2005 en las tres estaciones de su eje longitudinal (Tabla 7). Los oligoquetos son el grupo más representado, seguido de los quironómidos. La relación entre ambos grupos indica la clara dominancia de los oligoquetos; si bien su importancia relativa va disminuyendo hacia la cola. La presencia de esta fauna bentónica refleja el buen estado del embalse, que mantiene organismos propios de ambientes profundos en los que la diversidad es baja y las condiciones de oxígeno restrictivas. La comunidad es propia de sistemas oligotróficos con una dependencia mayor de los aportes de origen alóctono que de la producción propia del embalse. Este aporte nutricional es favorecido por la fluctuación del nivel del agua acompañada de procesos de sedimentación rápida.

Tabla 7. Densidad (ind $/ \mathrm{m}^{2}$ ) de macroinvertrbrados del bentos profundo en el embalse del Añarbe. Relación Q/O: quironómidos/oligoquetos. Density $\left(\mathrm{ind} / \mathrm{m}^{2}\right)$ of the deep benthic macroinvertebrates in the Añarbe reservoir. Q/O relation: Quironomidae/Oligochaeta.

\begin{tabular}{|c|c|c|c|c|c|c|}
\hline \multirow[b]{2}{*}{ TÁXONES } & \multicolumn{2}{|c|}{ PRESA } & \multicolumn{2}{|c|}{ CENTRO } & \multicolumn{2}{|r|}{ COLA } \\
\hline & $23 / 6 / 05$ & 8/11/05 & $23 / 6 / 05$ & $8 / 11 / 05$ & $23 / 6 / 05$ & 8/11/05 \\
\hline Nematoda & & & & & & \\
\hline Mermithidae & & & & 87 & & \\
\hline Annelida & & & & & & \\
\hline Oligochaeta & 1087 & 1391 & 1783 & 2174 & 1174 & 1261 \\
\hline Helobdella sp. & 43 & & 43 & & & \\
\hline Hydrachnidae & & & & & & \\
\hline Hydrachna sp. & & & & & 43 & 87 \\
\hline Crustacea & & & & & & \\
\hline Ostracoda & & 43 & & 522 & & \\
\hline Insecta (Chironomidae) & & & & & & \\
\hline Tanypodinae & 130 & & 391 & 87 & 130 & 174 \\
\hline Tanytarsini & & & 87 & & 43 & 43 \\
\hline Chironomus plumosus & & & & & 174 & 956 \\
\hline Mollusca & & & & & & \\
\hline Physidae & & & & 43 & & \\
\hline Pisidium sp. & & & 130 & 43 & 217 & 43 \\
\hline TOTAL (ind/m²) & 1260 & 1434 & 2434 & 2956 & 1781 & 2564 \\
\hline Relación $Q / O$ & 0.12 & 0 & 0.27 & 0.04 & 0.29 & 0.93 \\
\hline
\end{tabular}


Tabla 8. Valoración del estado trófico en el embalse del Añarbe. Desde mayo de 2004 a febrero de 2005 (2004/2005) y desde junio de 2005 a enero de 2006 (2005/2006). Appraisal of the trophic state of the Añarbe reservoir. From May of 2004 to February of 2005 (2004/2005) and from June of 2005 to January of 2006 (2005/2006).

\begin{tabular}{|c|c|c|c|c|}
\hline & \multicolumn{2}{|c|}{$2004 / 05$} & \multicolumn{2}{|c|}{$2005 / 06$} \\
\hline \multicolumn{5}{|l|}{ OCDE (1982) } \\
\hline PT $(\mu \mathrm{g} / \mathrm{l})$ & 27.01 & Mesotrófico & 11.82 & Mesotrófico \\
\hline media fótica Chl a $(\mu \mathrm{g} / \mathrm{l})$ & 0.90 & Ultraoligotrófico & 0.54 & Ultraoligotrófico \\
\hline Chl a $\max (\mu \mathrm{g} / \mathrm{l})$ & 2.40 & Ultraoligotrófico & 0.56 & Ultraoligotrófico \\
\hline Secchi (m) & 7.40 & Oligotrófico & 8.50 & Oligotrófico \\
\hline Secchi min $(m)$ & 4.90 & Oligotrófico & 5.80 & Oligotrófico \\
\hline \multicolumn{5}{|l|}{ EPA (1976) } \\
\hline PT $(\mu \mathrm{g} / \mathrm{l})$ & 27.01 & Eutrófico & 11.82 & Mesotrófico \\
\hline $\mathrm{n}^{\circ}$ cel algales $/ \mathrm{ml}$ & 90.90 & Oligotrófico & 275 & Oligotrófico \\
\hline Chl a max $(\mu \mathrm{g} / \mathrm{l})$ & 2.40 & Oligotrófico & 0.56 & Oligotrófico \\
\hline \multicolumn{5}{|l|}{ Margalef (1983) } \\
\hline $\mathrm{PT}(\mu \mathrm{g} / \mathrm{l})$ & 27.01 & Eutrofia avanzada & 11.82 & Eutrofia moderada \\
\hline $\mathrm{n}^{\circ}$ cel algales $/ \mathrm{ml}$ & 90.90 & Eutrofia moderada & 275 & Eutrofia moderada \\
\hline media fótica Chl a $(\mu \mathrm{g} / \mathrm{l})$ & 0.90 & Eutrofia moderada & 0.54 & Eutrofia moderada \\
\hline $\operatorname{Secchi}(\mathrm{m})$ & 7.40 & Eutrofia moderada & 8.50 & Eutrofia moderada \\
\hline \multicolumn{5}{|l|}{ Carlson (1974) } \\
\hline TSI secchi & 31.20 & Oligotrófico & 29.16 & Oligotrófico \\
\hline TSI Chl a fótica & 29.80 & Oligotrófico & 24.55 & Oligotrófico \\
\hline TSI PT fótico & 55.60 & Eutrófico & 39.75 & Mesotrófico \\
\hline TSI medio & 38.87 & Mesotrófico & 31.15 & Oligotrófico \\
\hline
\end{tabular}

Así, la comunidad trófica aparece dominada por los recolectores a lo largo de todo el eje longitudinal del embalse, si bien los detritívoros, oligoquetos fundamentalmente, pierden peso respecto a los filtradores a medida que desciende la profundidad hacia la cola. Entre estos últimos destacan los quironómidos Tanytarsini y Chironomus plumosus y el molusco bivalvo Pisidium sp. Cabe mencionar también la presencia significativa de depredadores como es el caso del quironómido Tanypodinae.

La microbiología de las aguas del embalse no presenta problemas, como corresponde a una cuenca poco humanizada. La densidad de coliformes totales es indicativa en todo el eje del embalse de una calidad de agua tipo A2 (entre 50$5000 \mathrm{ufc} / 100 \mathrm{ml}$ ) para producción de agua potable (R.D. 927/1988, de 29 de julio).

\section{Estado trófico}

La estima del estado trófico del embalse se ha realizado con los valores medios anuales de la zona de la presa, ya que son las condiciones más similares a un lago y el estado trófico de esta zo- na es el que va a afectar directamente a la calidad del agua de abastecimiento.

El estado trófico del embalse de Añarbe es en general bueno, indicando niveles de oligotrofia para la mayor parte de los índices excepto para las concentraciones de fósforo total, que según el índice considerado se incluye en la categoría de mesotrofia o eutrofia (Tabla 8). La concentración media de fósforo total es menor en el ciclo anual 2005/2006, que en el periodo anterior, aún así el nivel medio de fósforo total indica una ligera mesotrofia.

Estos resultados son coincidentes con los presentados en el informe de Basoinsa (1994) que señalan la misma problemática trófica debida al fósforo en los años 1991, 1992 y 1993.

\section{Potencial ecológico}

El mayor porcentaje de variación del volumen respecto al volumen máximo supera el límite propuesto para el MPE en los dos años estudiados. Por lo tanto, las características hidromorfológicas, en este caso la variación del 
Tabla 9. Valoración de los diversos indicadores analizados en el embalse de Añarbe para la estima del Potencial Ecológico; A: (enero 2004-febrero 2005) y B: (junio 2005-enero 2006). Se consideran los parámetros medios en la zona de la presa del embalse. Appraisal of the indicators analyzed in the Añarbe reservoir to estimate the ecological potential; A: (January 2004- February 2005) and B: (June 2005- January 2006). The average parameters in the zone of the dam of the reservoir are considered.

\begin{tabular}{|c|c|c|c|c|c|c|}
\hline \multirow{3}{*}{ Parámetro } & \multicolumn{2}{|c|}{ Añarbe } & \multicolumn{4}{|c|}{ Valoración } \\
\hline & \multicolumn{2}{|c|}{ Valor } & \multirow{2}{*}{ Parámetro } & \multirow{2}{*}{ Elemento } & \multirow{2}{*}{ Indicador } & \multirow{2}{*}{$\begin{array}{c}\text { Potencial Ecológico } \\
\text { provisional }\end{array}$} \\
\hline & A & $\mathrm{B}$ & & & & \\
\hline Densidad algal total, media anual (cel/ml) & 90.90 & 275 & 5 & \multirow{4}{*}{5} & \multirow{4}{*}{5} & \multirow{9}{*}{3.3} \\
\hline Chl a. media anual fótica $(\mu \mathrm{g} / \mathrm{l})$ & 0.90 & 0.54 & 5 & & & \\
\hline Chl a máxima anual ( $\mu \mathrm{g} / \mathrm{l})$ & 2.40 & 0.56 & 5 & & & \\
\hline Cianobacterias, máximo anual (cel/ml) & 0.00 & 303 & 5 & & & \\
\hline Secchi. media anual (m) & 7.40 & 8.50 & 4 & 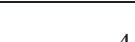 & \multirow{5}{*}{3.3} & \\
\hline Secchi. mínimo anual (m) & 4.90 & 5.80 & 4 & 4 & & \\
\hline Oxígeno hipolimnético & & & & & & \\
\hline medio durante estratificación (mg/l) & 4.52 & 4.58 & 3 & 3 & & \\
\hline Fósforo total, media anual ( $\mu \mathrm{gP} / \mathrm{l})$ & 27.01 & 11.82 & 3 & 3 & & \\
\hline
\end{tabular}

volumen por encima de un $20 \%$ del máximo, supone una alteración hidromorfológica que impide alcanzar el MPE. La oscilación del volumen es inherente al uso para el que fue construido el embalse del Añarbe y no impide que pueda tener un buen potencial ecológico, sino únicamente que éste no será el máximo posible.

La asignación provisional del potencial ecológico se realiza teniendo en cuenta la metodología descrita anteriormente y se resume en la Tabla 9. La aproximación al cálculo del potencial ecológico del embalse del Añarbe lo señala como Bueno, aunque con una valoración próxima a Moderado, debido a que la concentración media anual de fósforo total se encuentra dentro del rango de la mesotrofia y a que la cantidad de oxígeno hipolimnético se encuentra también dentro de la clase moderada. La puntuación final en esta primera aproximación al cálculo del potencial ecológico del embalse del Añarbe es de 3.3, que podría ser considerada como de referencia para su tipo.

\section{AGRADECIMIENTOS}

Agradecemos el trabajo realizado por Encarna Zafra y la Dra. Marina Aboal de la Universidad de Murcia por la identificación de las especies fitoplanctónicas; y a Santiago Robles de la empresa CIMERA S.A. y al Dr. Miguel Alonso por la identificación del zooplancton. Gracias también al personal del laboratorio de AGASA por facilitar todos los medios necesarios para la realización de los muestreos de campo, así como al personal de la presa del Añarbe por el apoyo prestado. Finalmente, los trabajos de campo no hubieran sido posibles sin la colaboración de Jesús Ángel Arrate ni de Eva López (Anbiotek S.L.).

\section{BIBLIOGRAFÍA}

AGÈNCIA CATALANA DE L'AigUA. 2003. Caracterització i propostes d'estudi dels embassaments catalans segons la Directiva 2000/60/CE del Parlament Europeu. 212 pp.

AGÈNCIA CATALANA DE L'AIGUA. 2006. Protocol d'avaluació del potencial ecològic dels embassaments. ECOEM. 47 pp.

APHA-AWWA-WPCF. 1992. Métodos normalizados para el análisis de aguas potables y residuales. 17th Edición. Ed. Días de Santos, S.A. Madrid. $1550 \mathrm{pp}$.

BASOINSA, S. L. 1994. Estudio de propuesta de usos de la cuenca del embalse de Añarbe para salvaguardar las características de calidad del agua y vida útil del embalse. Fase I. $2^{a}$. Hidrología. Mancomunidad de Aguas del Añarbe-Añarbeko uren mankomunitatea. 29 pp.

CARLSON, R. E. 1974. A trophic state index for lakes. Limnol. Oceanogr., 23: 361-369.

CATALÁN, J. 1987. Limnologia de l'estany Re- 
dó (Pirineu Central). Tesis Doctoral. Universidad de Barcelona. 230 pp.

CHORUS, I. \& J. BARTRAM. 1999. Toxic Cianobacteria in water. A guide to their public health consequences, monitoring and management. Word Health Organization (WHO). London. E \& FH Spon. 416 pp.

DOCE. 2000. DIRECTIVA 2000/60/CE del Parlamento Europeo y del Consejo de 23 de octubre de 2000. Diario Oficial de las Comunidades Europeas, Serie L $\mathrm{n}^{\circ} 327$, de 22 de Diciembre de 2000. $72 \mathrm{pp}$.

GOBIERNO VASCO. 2002. Caracterización de las masas de aguas superficiales de la CAPV. Tomo 3. Embalses. Dpto. Ordenación del Territorio y Medio Ambiente. Dirección de Aguas. 197 pp.

HÄKANSON, L. 1981. A manual of Lake Morphometry. Springer-Verlag Ed. Berlin. 78 pp.

HUTCHINSON, G. E. 1957. A treatise on limnology. Vol. I. Geography, physics and chemistry. John Wiley \& Sons, New York, NY. 1015 pp.

INFRAESTRUCTURA \& ECOLOGÍA S.L. 2003. Seguimiento de la calidad de las aguas en embalses de zona sensibles. Documento de Síntesis. MMA. CHE. Comisaría de aguas. 45 pp.

INFRAESTRUCTURA \& ECOLOGÍA S.L. 2006. Memoria. Ejecución de trabajos relacionados con los requisitos de la Directiva Marco (2000/60/CE) en el ámbito de la Confederación Hidrográfica del Ebro referidos a: Elaboración del registro de zonas protegidas, Determinación del potencial ecológico en embalses, Desarrollo de programas específicos de investigación. MMA. CHE. Dirección General del Agua. 88 pp.

INTERNATIONAL STANDARD, ISO 9391:1993 (E). Water quality-Sampling in deep waters for macroinvertebrates-Guidance on the use of colonization, qualitative and quantitative samplers. $13 \mathrm{pp}$.

JRC Report EUR 14563. PREMAZZI, G. \& CHIADUANNI, G. 1992. Ecological quality of Surface Water. Comisión of the European Communities, $124 \mathrm{pp}$.

LARUMBE, M. I. 1991. Estudio de las variables que influyen sobre la calidad de las aguas del embalse de Añarbe. Tesis Doctoral. Universidad del País Vasco/Euskal Herriko Unibertsitatea. Fac. Químicas. Donostia-San Sebastián. 258 pp.

MARGALEF, R. 1983. Limnología. Ed. Omega. Barcelona. $1010 \mathrm{pp}$.

OCDE. 1982. Eutrophisation des eaux. Métodes de surveillance, d'evaluation et de lutte. Paris. 164 pp.

PÉREZ-BOTE, J. L., R. MORÁN, R. ROSO \& A. J. ROMERO. 2005. Craspedacusta sowerbii, la medusa que vino de Asia. Quercus, 236: 22-25.

REAL DECRETO 927/1988, de 29 de julio. Reglamento de la Administración Pública del Agua y de la Planificación Hidrológica, en desarrollo de los títulos II y III de la Ley de Aguas. B.O.E. núm. 209: 26412-16425, de 31 de agosto de 1988.

U. S. EPA.1976. Quality Criteria for water. The red book. Washington, D.C. (EPA 440/9-76-023, July, 1976). $534 \mathrm{pp}$.

UTERMÖHL, H. 1958. Zur Vervollkommnung der quantitativen Phytoplankton-Methodik. Mitt. Int. Ver. Theor. Angew. Limnol., 9: 1-38.

WETZEL, R. G. 1981. Limnología. Ed. Omega. Barcelona. $679 \mathrm{pp}$.

WFD CIS Guidance Document $\mathrm{N}^{\circ}$ 4. 2003. Identification and Designation of Artificial and Heavily Modified Waterbodies. Published by the Directorate General Environment of the European Comisión, Brussels, ISBN No 92-894-5124-6, ISSN $\mathrm{N}^{\mathrm{o}}$ 1725-107.

WFD CIS Guidance Document $\mathrm{N}^{\circ}$ 10. 2003. Rivers and Lakes-Typology, Reference Conditions and Classification Systems. Published by the Directorate General Environment of the European Comisión, Brussels, ISBN No 92-894-5614-0, ISSN $\mathrm{N}^{\mathrm{o}} 1725-1087$.

WFD CIS Guidance Document $\mathrm{N}^{\mathrm{o}} 13.2005$. Overall Approach to the Classification of Ecological Status and Ecological Potential. Published by the Directorate General Environment of the European Comisión, Brussels, ISBN N 92-894-6968-4, ISSN $\mathrm{N}^{\mathrm{o}} 1725-1087$. 